\title{
Cambios en el uso y cobertura de la tierra entre 1948 y 2012 en el Humedal Nacional Térraba-Sierpe, Costa Rica
}

\author{
Land use and cover changes between 1948 and 2012 in the \\ Térraba-Sierpe National Wetlands, Costa Rica
}

\author{
Jéssica Francini Acuña-Piedra ${ }^{1}$ y Adolfo Quesada-Román ${ }^{2 *}$
}

\begin{abstract}
RESUMEN
El Humedal Nacional Térraba-Sierpe (HNTS) es el humedal más grande de Costa Rica, se localiza al SE del país entre las coordenadas geográficas $8^{\circ} 47^{\prime \prime}$ Latitud Norte y $83^{\circ} 38^{\prime \prime}$ Longitud Oeste. Está constituido por el delta Térraba-Sierpe, el cual está cubierto de una densa vegetación de manglar que ha sido modificada durante más de seis décadas hacia usos agrícolas de la tierra. El objetivo de este trabajo fue estudiar los cambios de coberturas y usos de la tierra que ha tenido el Humedal Nacional Térraba-Sierpe a partir de la interpretación de fotografías aéreas y una imagen satelital, además de corroboración de campo para entender las tendencias durante 64 años de análisis asociadas a patrones climáticos, geomorfológicos y antrópicos. Se determinó que la deforestación tuvo una pérdida periódica neta entre 1948 y 2012 de más de 2562.79 ha, con una pérdida anual neta de 40 ha por año. Este trabajo generó una línea base en el análisis de los cambios en los usos y coberturas de la tierra del humedal Térraba-Sierpe, cuantificando los impactos antrópicos causados a lo largo de más de 40 años en el manglar y zonas adyacentes, y puso en evidencia las buenas decisiones en legislación ambiental tomadas durante la década de 1990 para la protección de estos frágiles ecosistemas.
\end{abstract}

Palabras claves: Uso de la tierra, manglar, humedal, deforestación, impacto ambiental.

\begin{abstract}
The Térraba-Sierpe National Wetlands (HNTS) is the largest wetland in Costa Rica, located in the South-Eastern part of the country between the geographical coordinates $8^{\circ} 47^{\prime \prime}$ North Latitude and $83^{\circ} 38^{\prime}$ " West Longitude. It is comprised of the Térraba-Sierpe delta, which is covered with dense mangrove vegetation that has been modified for over six decades for agricultural land uses. This paper was aimed at studying land use and cover changes of the Térraba-Sierpe National Wetlands based on the interpretation of aerial photographs and a satellite image, as well as field corroboration to understand the trends during the 64 years of analysis associated with climatic, geomorphological, and anthropic patterns. A net periodic loss by deforestation was determined between 1948 and 2012 exceeding 2562.79 ha, with an annual net loss of 40 ha per year. This paper helped to generate a baseline in the analysis of land use and cover changes in the Térraba-Sierpe Wetlands, quantify the anthropic impact on the mangrove and adjacent areas over 40 years, and evidence the good decisions made in terms of environmental legislation during the 1990s for the protection of these fragile ecosystems.
\end{abstract}

Keywords: Land use, mangrove, wetlands, deforestation, environmental impact.

Proyecto Humedales, SINAC-GEF-PNUD-MINAE. Santo Domingo de Heredia. fran1993piedra@gmail.com. ${ }^{2} \mathrm{Cli}-$ matic Change and Climate Impacts, Institute for Environmental Sciences, University of Geneva, Boulevard CarlVogt 66, CH-1205 Geneva, Switzerland. adolfo.quesada@etu.unige.ch, adolfo.quesada@gmail.com*

Recibido: 19 de julio de 2017

Corregido: 5 de octubre de 2017

Aceptado: 9 de octubre de 2017

DOI: http://dx.doi.org/10.15359/revmar.9-2.1 


\section{INTRODUCCIÓN}

El sistema deltaico de TérrabaSierpe se ubica al sureste de Costa Rica, en el cantón de Osa, en el área protegida del Humedal Nacional Térraba-Sierpe (HNTS), el cual tiene una extensión de 26779 ha basado en el límite oficial del decreto de creación de Área Silvestre Protegida (Proyecto Humedales, 2015). El área de estudio se caracteriza por ser el humedal más grande del país y además está bordeado por dos grandes cauces como son el río Sierpe y el Grande de Térraba (Sánchez, 1986), y se considera que cuenta con aproximadamente el $43 \%$ de la vegetación de mangle del país (Mainardi, 1996). La ubicación del HNTS es en la provincia de Puntarenas, en el cantón de Osa, entre los distritos de Cortés, Palmar Norte y Sierpe (Fig. 1), entre las coordenadas geográficas $8^{\circ} 47^{\prime \prime}$ Latitud Norte y $83^{\circ}$ $38^{\prime}$ Longitud Oeste, sus límites son entre la Fila Brunqueña y la serranía de la Península de Osa en el valle de El Diquís (BIOMARCC-SINAC-GIZ, 2012). De acuerdo con Kappelle et al. (2002), el HNTS pertenece a un Área Silvestre Protegida, la cual fue creada en 1994 y se localiza en el Área de Conservación Osa (ACOSA).

El HNTS está constituido por una amplia planicie costera y un patrón de drenaje meándrico que conforma el delta Térraba-Sierpe, así como una cobertura densa de manglar (Scheffers \& Browne, 2010). Este tipo de vegetación de mangle se caracteriza por una alta fragilidad, la cual está asociada a las condiciones específicas que necesita para desarrollarse; requiere de medios acuáticos $\mathrm{y}$ salinos, así como de suelos fangosos e inestables para su crecimiento, además presenta órganos especiales de respiración y sostén, metabolismos adaptados a altas concentraciones de sal y viviparidad (Méndez \& Guzmán, 2006). Las características específicas del manglar, lo vuelve susceptible a cualquier cambio o modificación en el entorno, tanto por diferentes agentes naturales como los cambios en la temperatura, el viento, las mareas, las variaciones en la salinidad de las aguas, las modificaciones en los aportes de agua dulce, las precipitaciones, los sedimentos y principalmente aspectos geomorfológicos $\mathrm{y}$, también, por las actividades antrópicas como por el crecimiento urbano no controlado, el turismo indiscriminado, además del cambio en el uso de la tierra y la deforestación (Zaldívar et al. 2004).

El delta Térraba-Sierpe se ha enfrentado a cambios en los últimos 60 años en la cobertura del manglar, ya que se han desarrollado usos de la tierra dentro del HNTS que han desplazado el hábitat de mangle. Entre las actividades que sobresalen están: la siembra de arroz, la palma aceitera, el pasto para ganado, las salineras y la construcción de estanques para el cultivo de camarón, esto se debe a que son áreas planas o de baja pendiente y suelen ser suelos de origen sedimentario de alta productividad (Jiménez, 1993). Hasta 1996, con la publicación oficial de la Ley Forestal número 7575 se realizaron procesos de deforestación de los árboles de mangle para la producción y comercialización de carbón, madera y corteza para taninos, en la que se utilizaban los árboles del género Rhizophora. 


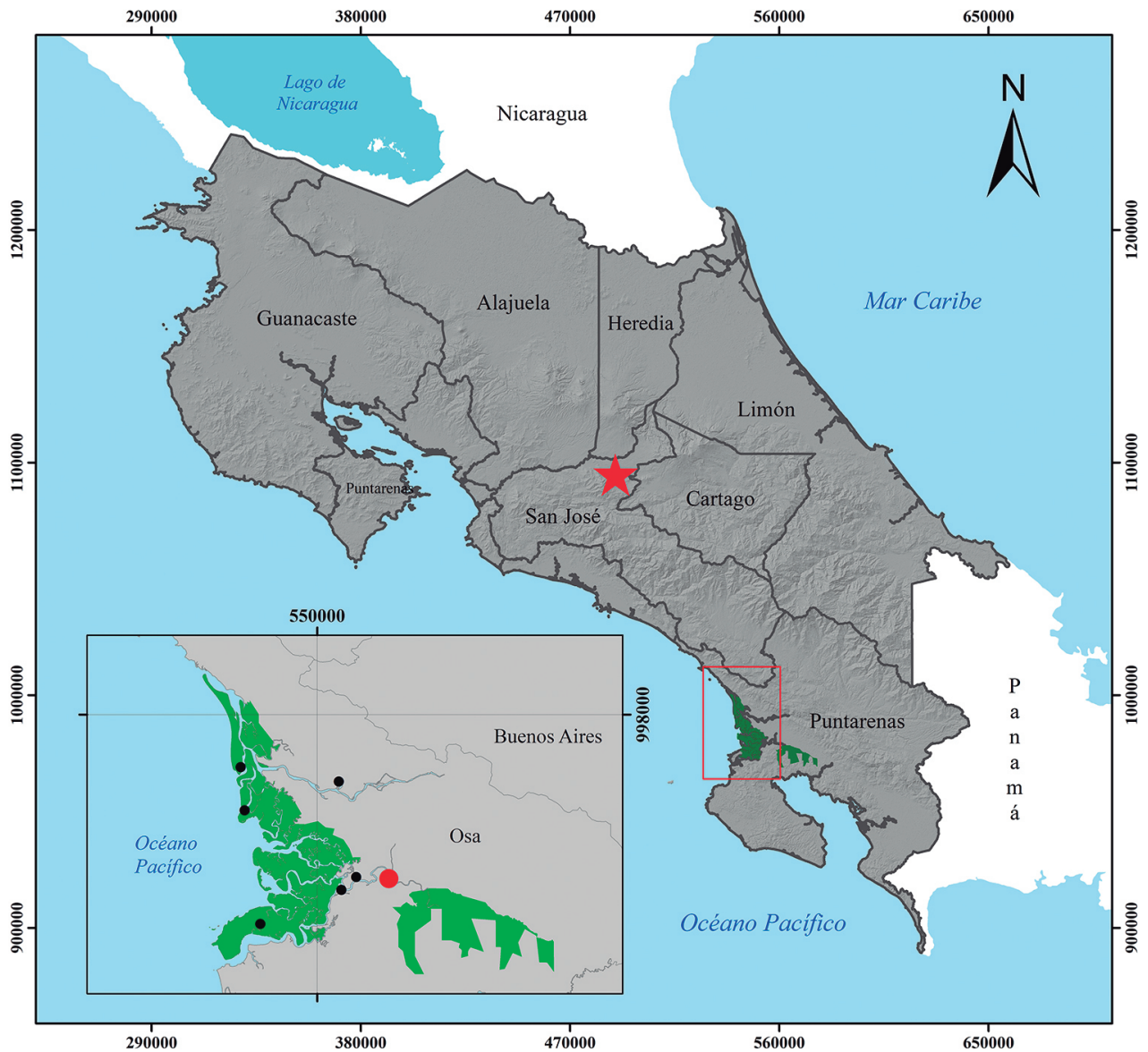

\section{Simbología}

† Capital San José

Poblado de Sierpe

Poblados HNTS

Humedal Nacional Térraba-Sierpe Área de Estudio

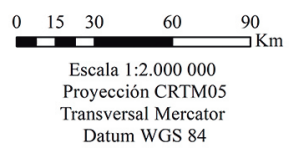

Fuente: Sistema Nacional de Áreas de Conservación, 2014 Instituto Geográfico Nacional Cartografía Escala 1:50.000

Atlas Cantonal, Instituto Tecnológico de Costa Rica, 2014

Fig. 1. Localización del Humedal Nacional Térraba-Sierpe: Osa, Costa Rica Fig. 1. Location of the Térraba-Sierpe National Wetlands: Osa, Costa Rica

También dentro del humedal se dan otras actividades como la extracción de moluscos, sobre todo la piangua, esta es una de las principales actividades de los habitantes de este sistema fluviomarino (Vargas, 2007).

En el pasado se han realizado diferentes estudios sobre la cobertura y diná- mica geomorfológica de este territorio y sus cambios ecológicos a través del tiempo (Barrantes \& Cerdas, 2015; Lizano et al. 2001; Mora, 2013; Ortiz-Malavassi, 2012). A pesar de que el HNTS ha experimentado cambios expansivos en la cobertura y uso de la tierra desde 1948, para el año 2012 se visualiza un proce- 
so de recuperación del área de mangle, esto se debe a que el área del humedal pasó a ser un área protegida para el año 1994 y sitio Ramsar en 1995 (Pizarro et al. 2004), lo cual prohíbe que se desarrollen actividades antrópicas dentro del humedal. El objetivo de este trabajo fue estudiar los cambios de coberturas y usos de la tierra que ha tenido el Humedal Nacional Térraba-Sierpe para entender las tendencias durante 64 años de análisis asociadas a patrones climáticos, geomorfológicos y antrópicos.

\section{MATERIALES Y MÉTODOS}

\section{Procesamiento y análisis de imágenes aéreas}

El primer proceso del trabajo consistió en la georreferenciación de tres fotografías aéreas de los años 1948, 1972 y 1992, las cuales tienen una escala 1:50.000, 1:20.000 y 1:60.000 respectivamente, estos insumos fotográficos fueron suministrados por el Instituto Geográfico Nacional de Costa Rica (IGN); además se utilizó una imagen satelital Rapid Eye del año 2012 con resolución de pixel de $5 \mathrm{~m}$ obtenida del Centro Nacional de Alta Tecnología de Costa Rica (CeNAT). Para cada año se delimitó el área de estudio mediante la fotointerpretación en el software Arc Map versión 10.3.1 (ESRI, 2010), para el caso de las fotografías aéreas se digitalizó a escala 1:2.000 y para la imagen satelital 1:3.000, con este procesamiento se lograron obtener los diferentes usos de la tierra del HNTS para los cuatro años de análisis y visualizar los cambios en sus extensiones durante seis décadas de estudio, los cuales fueron verificados en campo. Al tener diferentes escalas fue necesario determinar las diferencias que presentaron cada una de las fotografias, ya que al comparar las extensiones es importante conocer el margen de error; según Salitchev (1979) para la escala 1:50.000 el margen de error de la fotografía es correspondiente a $50 \mathrm{~m}$, para la escala 1:20.000 presenta $20 \mathrm{~m}$, en el caso de la fotografía aérea a 1:60.000 tiene $10 \mathrm{~m}$ y en el caso de la imagen satelital es de $25 \mathrm{~m}$.

\section{Digitalización de la cobertura y uso de la tierra}

El desarrollo de esta etapa incluyó la clasificación de usos y coberturas de la tierra, mediante la fotointerpretación en el software Arc Map versión 10.3.1 (ESRI, 2010), las fotografías aéreas se digitalizaron a escala 1:2.000 y la imagen satelital a 1:3.000. En este proceso se definieron las clases de cobertura apoyadas en la metodología de la Leyenda Corine Land CoverCosta Rica (CLC-R) (Rosales, 2016), la cual consta de 61 clases, de las cuales nueve de ellas, se identificaron en el área de estudio. Se localizaron seis usos de la tierra, los cuales son: arroz, pastos limpios y arbolados, espacios naturales (que incorporan todas aquellas áreas que no presentan ningún tipo de vegetación manglárica y que se encuentran intervenidas tanto de forma natural como antrópica. Se incluyen todas las áreas con charral, pastos limpios y toda aquella vegetación no identificada taxonómicamente pero no manglárica, según los espectros), palma aceitera, tierras desnudas o degradadas y estanques de maricultura (Cuadro 1); asimismo, se identificaron cinco clases de coberturas naturales: 
manglar, zonas pantanosas, arenas, lagunas costeras y playas (Cuadro 1).

\section{Análisis de datos de deforestación}

Para establecer cuál fue la pérdida de la cobertura de mangle en el HNTS con respecto a la expansión de las actividades de uso de la tierra, se realizó un análisis de pérdida neta, periódica y la tasa de deforestación (Duarte et al. 2015). La Pérdida Periódica Neta (PNN) se refiere a la deforestación en un tiempo determinado, $P N N$ : $C M_{1}-C M_{2}$.

Donde, $C M_{1}$ corresponde a la cobertura de mangle para los primeros años de análisis y $C M_{2}$ se refiere a la cobertura en el año final del periodo analizado.

La Pérdida Periódica Anual Neta (PPAN) se obtuvo por promedio, el cual determina cuánta cobertura forestal de mangle desapareció por año, donde se aplica la fórmula de Duarte et al. (2015), PPAN=PPN/t.

Donde, $P P N$ corresponde a los datos obtenidos en la pérdida periódica neta y $t$ es el tiempo de estudio entre los años comparados.

La Tasa de Deforestación (D) se refiere a la pérdida de cobertura vegetal para ser sustituida por otra actividad de uso de la tierra (FAO, 2001), donde

Cuadro 1. Definiciones de las clases utilizadas en los usos y coberturas de la tierra. Fuente: Rosales (2016)

Table 1. Definition of classes used in land use and cover. Source: Rosales (2016)

\section{CLASE}

Usos de la tierra

Arroz

Palma Aceitera

Pastos Limpios

Pastos Arbolados

Tierras Desnudas o

Degradadas

Estanques para acuicultura y maricultura

Cobertura de la tierra

Playas y Arenas

Zonas Pantanosas

Manglar

Lagos o Lagunas

\section{DEFINICIÓN}

Planta Oriza sativa de la familia Poaceae, se siembra en superficies planas o ligeramente onduladas, en un rango de 0 a $200 \mathrm{msnm}$.

Palma aceitera (Elaesis guineensis), planta perenne de tronco solitario y hojas pinnadas.

Tierras ocupadas por pastos, sin presencia de maleza. Incluyen pastos de corta, ensilaje y heno.

Coberturas que incluyen pastos con presencia de árboles que superan los 5 metros de altura, se encuentran dispersos con presencia de entre un $15 \%$ a un $30 \%$ del área.

Superficie de terreno desprovisto de vegetación, debido a procesos naturales o antrópicos.

Cuerpos de agua destinados a la cría de crustáceos, moluscos y peces.

Terrenos bajos y planos constituidos por suelos arenosos y pedregosos.

Tierras planas que permanecen inundadas durante todo el día o la mayor parte del año.

Ecosistema dominado por grupos de especies pantropicales, arbóreas y arbustivas; incluye la Negraforra (Acrosticum aerum).

Superficies o depósitos de agua naturales de carácter abierto o cerrado, que pueden estar conectados o no a un río o al mar. 
se aplicó la fórmula de la FAO citada por Duarte et al. (2015), \% D: $\left(A_{2} / A_{p}\right)$ $\wedge\left(1 / t_{2}-t_{1}-1\right)$. Donde:

$A_{I}$ : área inicial de bosque de mangle (ha)

$A_{2}$ : área final de bosque de mangle (ha)

$t_{1}$ : año inicial

$t_{2}$ : año final.

\section{RESULTADOS Y DISCUSIÓN}

\section{Cambios en el uso y cobertura de la tierra, periodo de 1948 al 2012}

En cada periodo comparado se obtuvieron diferentes extensiones, tanto para la cobertura de mangle como para los distintos usos de la tierra, se evidencia que el HNTS enfrentó diversos procesos históricos que influyeron en la dinámica de la cobertura y los usos de la tierra, causando periodos de deforestación y en algunos momentos de recuperación de la vegetación de mangle. Los resultados para el primer año de análisis (1948) se caracterizaron por ser la época con más vegetación de mangle con 11256.23 ha que equivalen a un $73 \%$ del área del manglar; seguida por las zonas pantanosas, las cuales se componen de vegetación asociada a humedales (Acrostichum aureum), por lo que abarca 3019.91 ha que correspondieron a un 19\%; al sumarse con la categoría de mangle se obtuvo que la cobertura natural era de 14276.14 ha (Cuadro 2) que equivalen a un $92 \%$ del terreno (Fig. 2).

Se obtuvieron otras clases menos dominantes como las áreas sin infor- mación con 862.99 ha que corresponden a las zonas con nubosidad, así como también a vacíos de información con respecto a las fotografías aéreas, también se encuentran las áreas de playas y arenas con 248.59 ha. Se identificaron algunos usos de la tierra dentro del área del humedal, entre ellos, los pastos arbolados con 7.72 ha, las tierras desnudas y degradadas que cubren 18.73 ha, así como los pastos limpios y arbolados y su asociación con los espacios naturales que abarcan $31.96 \mathrm{ha}$, lo que se traduce a más de 58 ha de desarrollo agropecuario para la época (Fig. 2). Para este periodo la distribución del uso de la tierra se encontró en los sectores del humedal cercanos a las comunidades del distrito de Sierpe, donde se localizan algunos parches de pastos y también se desarrollaron usos hacia la zona de boca Zacate, donde se ubican áreas de pastos con árboles y terrenos descubiertos (Fig. 3). Los usos de la tierra solamente representaron un $1 \%$ del área del humedal, lo que sugiere un desarrollo incipiente de las actividades agropecuarias.

Para 1948 predominó la cobertura de mangle y representó más del $90 \%$ de terreno, los usos de la tierra eran de pocas extensiones y se identificaron pastos y espacios naturales, esta baja expansión agraria se relacionó con la poca población que había para este año en el cantón de Osa, donde no sobrepasaban los 2000 habitantes (Hernández, 1985). Para esa época las actividades económicas para la zona se basaban en una limitada agricultura de subsistencia de cultivo de granos básicos, árboles fru- 
Cuadro 2. Resultados de la cobertura de bosque de mangle y no mangle para el Humedal Nacional Térraba-Sierpe, en los años 1948, 1972, 1992 y 2012

Table 2. Results of mangrove and non-mangrove forest cover for the Térraba National Sierpe Wetlands during 1948, 1972, 1992 and 2012

\begin{tabular}{cccc}
\hline Año & $\begin{array}{c}\text { Cobertura de bosque } \\
\text { Mangle (ha) }\end{array}$ & $\begin{array}{c}\text { Cobertura de no bosque } \\
\text { Mangle (ha) }\end{array}$ & Total (ha) \\
\hline $\mathbf{1 9 4 8}$ & 14276.14 & 1169.99 & 15446.13 \\
$\mathbf{1 9 7 2}$ & 12817.63 & 1627.72 & 14445.35 \\
$\mathbf{1 9 9 2}$ & 11259.46 & 3241.08 & 14500.54 \\
$\mathbf{2 0 1 2}$ & 11713.35 & 2453.25 & 14166.80 \\
\hline
\end{tabular}

Fuente: Adaptado a partir de Duarte et al. (2015).

tales, la cría de animales (aves de corral, cerdos y ganado vacuno), la caza y la pesca (Royo, 2004) y la poca accesibilidad para llegar al humedal dificultaba el asentamiento y desarrollo agropecuario.

Durante los siguientes 24 años posteriores a 1948 hubo sustanciales cambios en el paisaje del HNTS, en el año 1972 la cobertura de mangle decrece y pasa a cubrir un $71 \%$, con una disminución de 1000 ha en comparación con 1948 (Cuadro 2). También se da un decrecimiento de las zonas pantanosas, pasan a cubrir un $18 \%$ del terreno, que se traduce en una pérdida de 391.83 ha con respecto a 1948; por lo tanto, el total de superficie ocupada por la vegetación del humedal es de 12817.63 ha que equivalen a un $89 \%$ del área total (Fig. 2). Asimismo, los usos de la tierra aumentaron y pasaron a ocupar más de 400 ha que equivalen a un $3 \%$ de la superficie del humedal, los usos identificados son los pastos limpios y arbolados que cubrían un área de 227.17 ha, los espacios naturales con una extensión de 185.15 ha, las tierras desnudas o degradadas que representan 0.74 ha y una nueva actividad, el cultivo de arroz, con una expansión de 3.92 ha. Se digitalizaron áreas de pequeñas lagunas costeras, arenas y playas, el análisis espacial continuó identificando faltantes de información (Fig. 4).

La distribución de los cambios en el uso de la tierra se dio en el distrito de Sierpe y Puerto Cortés, con el desarrollo de plantaciones de arroz cerca del límite del humedal y con algunos sectores sobrepasando lo que actualmente es el área protegida del humedal. Los pastos limpios ocupan un área considerable ubicada en comunidades como Cantarana y otros sectores del distrito de Sierpe cercano al manglar. En los poblados de boca Brava y boca Chiquita se aprecian otros usos como espacios naturales y terrenos degradados (Fig. 4). Por lo tanto, en estos 24 años, la situación en la cobertura de la tierra en el humedal cambió y la vegetación de mangle disminuyó en más de 1400 ha, esta pérdida de cobertura de mangle se relaciona con el incremento de las actividades del uso de la tierra, donde se identificó un aumento de actividades agrarias como el cultivo de arroz, pastos 

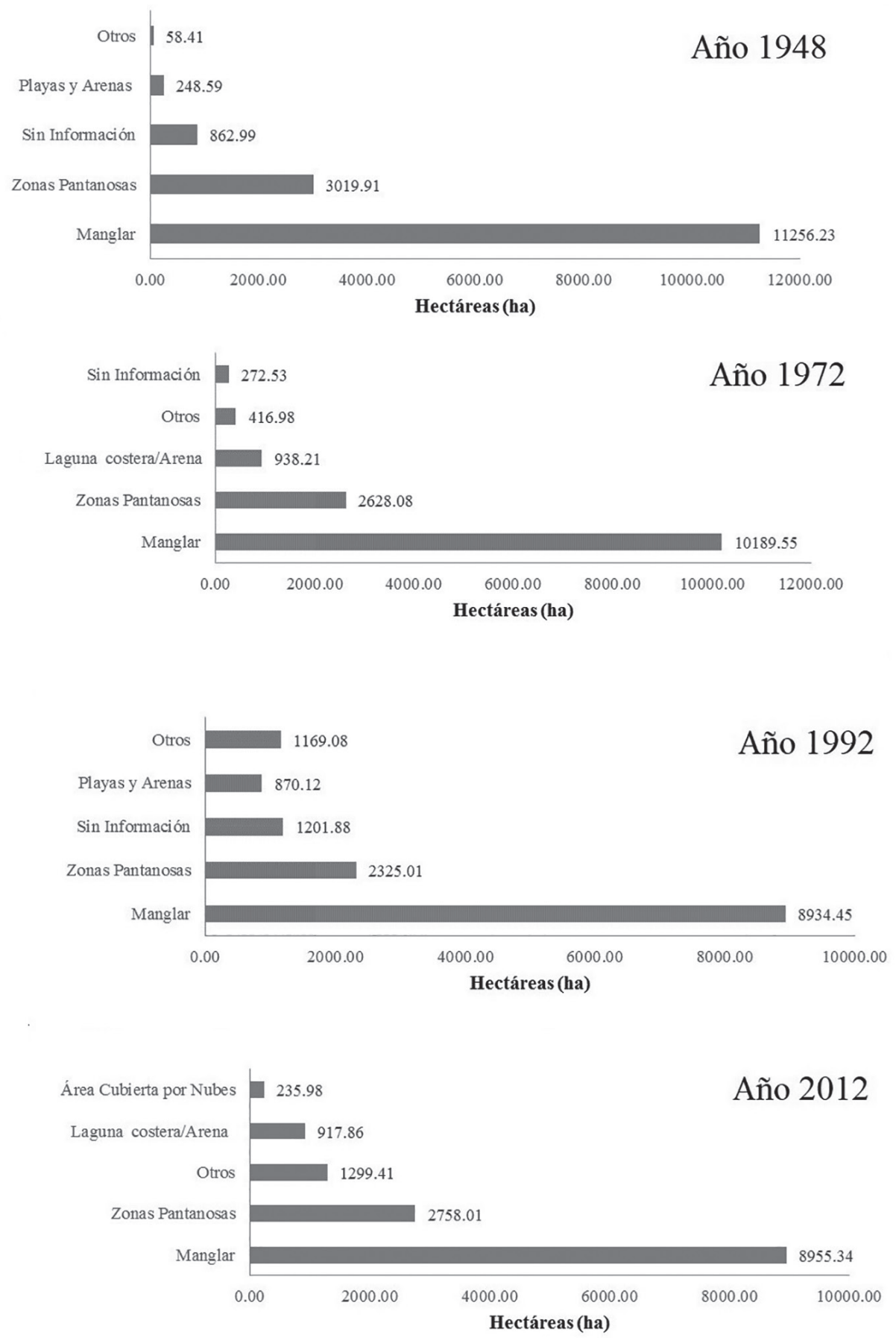

Fig. 2. Datos de los usos y coberturas de la tierra en el Humedal Nacional TérrabaSierpe para los años 1948, 1972, 1992 y 2012

Fig. 2. Data of land use and cover in the Térraba-Sierpe National Wetlands for years $1948,1972,1992$ and 2012 


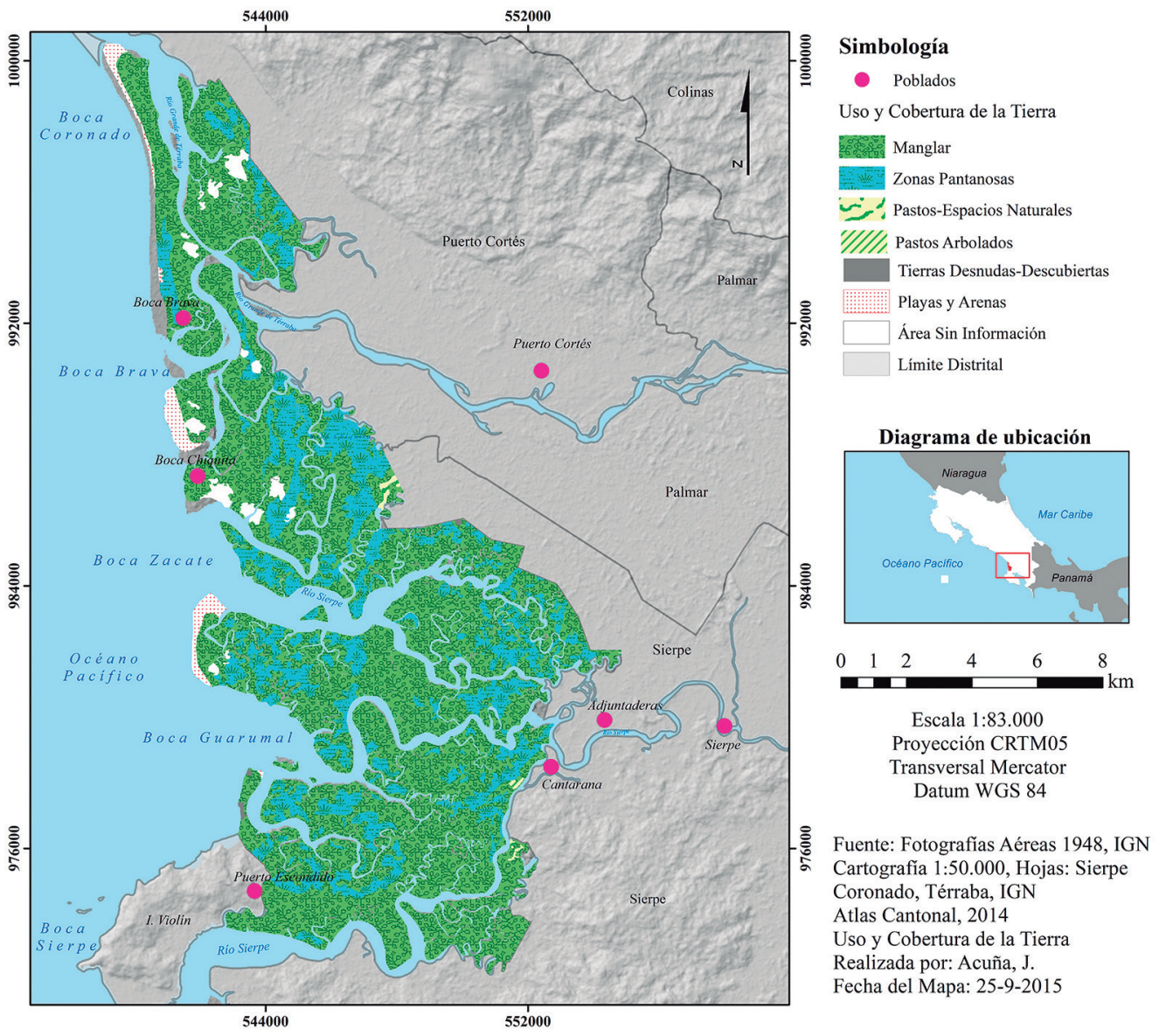

Fig. 3. Uso y cobertura de la tierra en el Humedal Nacional Térraba-Sierpe, año 1948 Fig. 3. Land use and cover in the Térraba-Sierpe National Wetlands, 1948

limpios y arbolados, espacios naturales, tierras desnudas o degradadas. Este incremento de los usos de la tierra se vincula al aumento poblacional que se dio en el cantón de Osa, atribuidos a los procesos históricos relacionados con el establecimiento de varias plantaciones de monocultivos como banano y las primeras plantaciones de piña y palma aceitera en el año 1964 (Cedeño et al. 2010), que fueron impulsoras de población debido a las fuentes de empleo. Los procesos de cambio que generaron las actividades de usos de la tierra y los naturales tuvieron influencia en la pérdida de mangle, ya que los cambios morfológicos en relación con la dinámica erosiva generaron que el humedal perdiera gran parte de su terreno en sectores como boca Zacate, Brava y Chiquita, que se ven afectados por el lavado de material, donde gran parte del terreno erosionado estaba cubierto de vegetación de mangle (AcuñaPiedra \& Quesada-Román, 2016). 


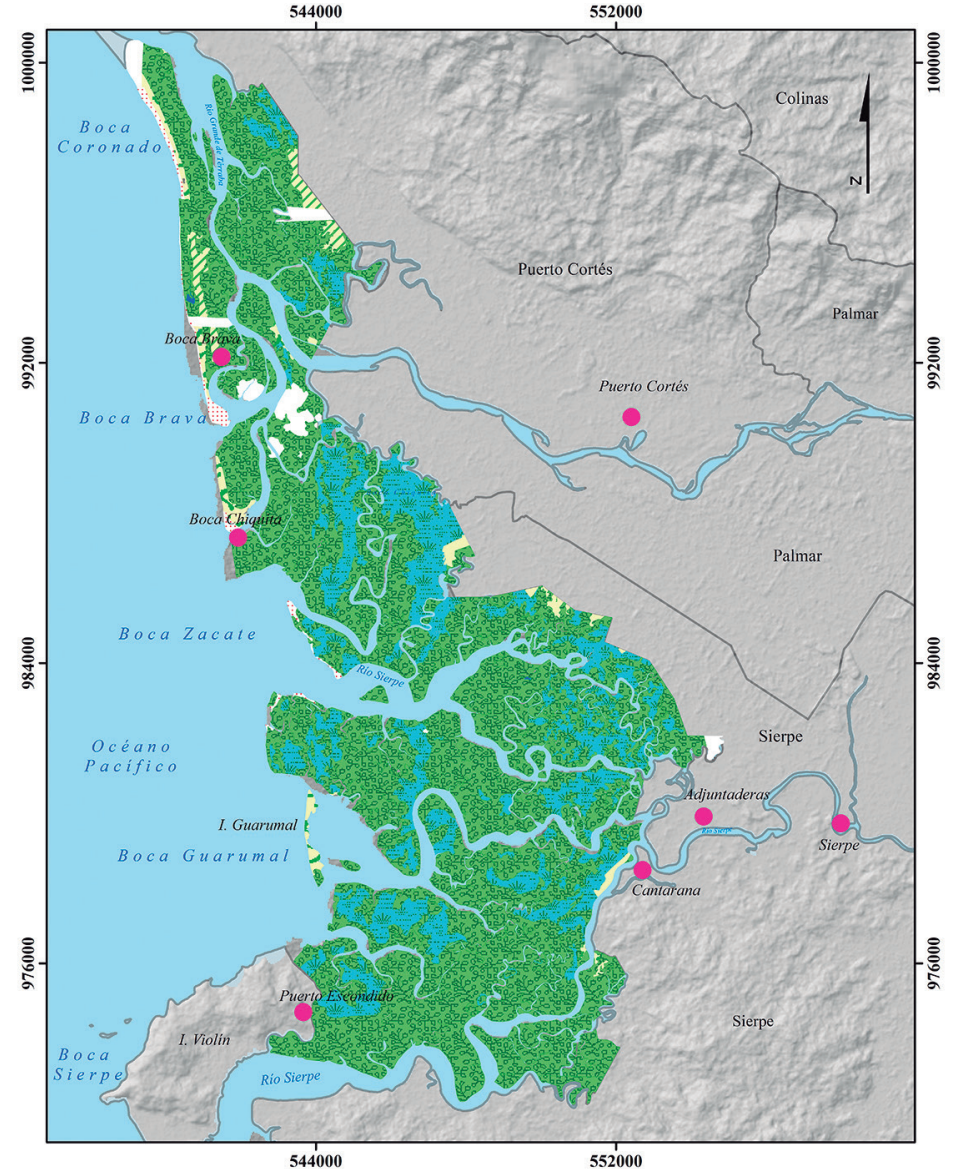

\section{Simbología}

- Poblados

Uso y Cobertura de la Tierra

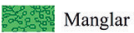

Zonas Pantanosas

X*. Pastos-Espacios Naturales

UIII/, Pastos Arbolados

Pastos Limpios

4.4.4. Arroz

Laguna Costera

Tierras Desnudas-Descubiertas

II: : Playas y Arenas

Área Sin Información

Límite Distrital

Diagrama de ubicación
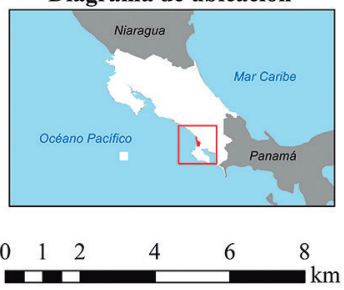

Escala 1:83.000

Proyección CRTM05

Transversal Mercator

Datum WGS 84

Fuente: Fotografias Aéreas 1972, IGN Cartografia 1:50.000, Hojas: Sierpe Coronado, Térraba, IGN Atlas Cantonal, 2014

Uso y Cobertura de la Tierra Realizada por: Acuña, J.

Fecha del Mapa: 25-9-2015

Fig. 4. Uso y cobertura de la tierra en el Humedal Nacional Térraba-Sierpe, año 1972 Fig. 4. Land use and cover in the Térraba-Sierpe National Wetlands, 1972

Durante el siguiente periodo que comprende 20 años de estudio entre 1972 y 1992, la vegetación del humedal siguió presentando un decrecimiento ocupando el bosque de mangle 8942 ha que corresponden a un $62 \%$ del área, y las zonas pantanosas pasan a ocupar más de 2324 ha que se traducen en un $16 \%$ de terreno, por lo que al sumarse corresponde a $11 \quad 259.66$ ha, esto representa una disminución de más de 1500 ha (Cuadro 2). Las actividades de los usos de la tierra siguen en aumento, su extensión crece en más de 700 ha, se identificaron espacios naturales que tenían una extensión de 671.11 ha, los pastos limpios y con árboles cubrían 315.68 ha, el cultivo de arroz abarcaba 23.53 ha, el terreno descubierto tenía 30.70 ha y se presenta el desarrollo de una nueva actividad, el establecimiento de estanques de maricultura con un área de 128.16 ha (Fig. 2). La distribución 


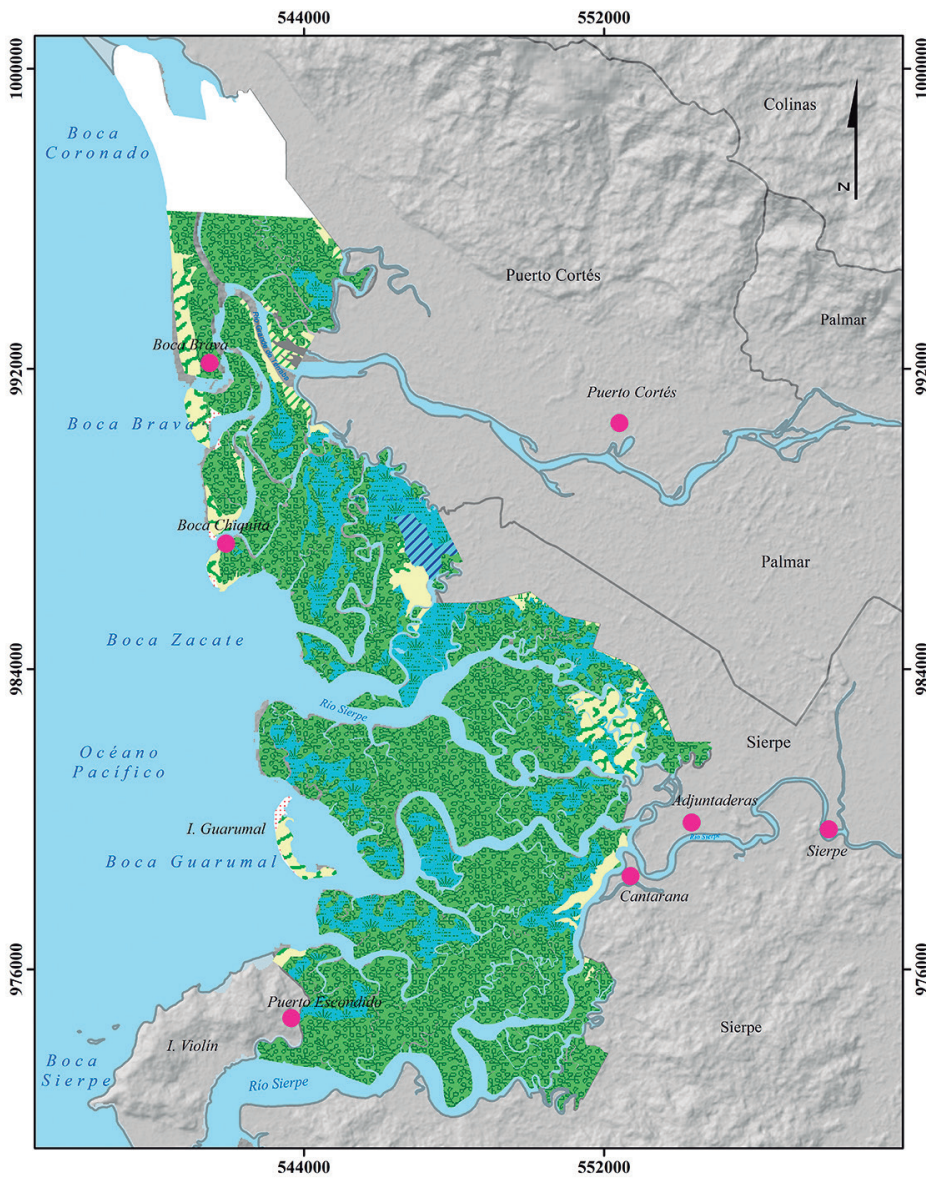

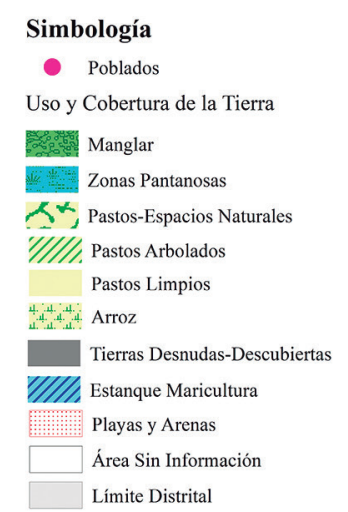

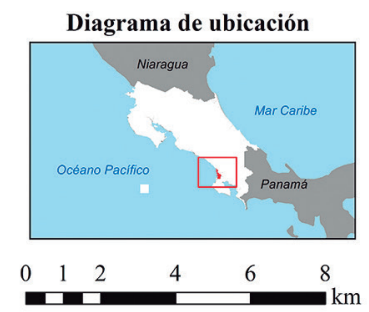

Escala 1:83.000 Proyección CRTM05 Transversal Mercator Datum WGS 84

Fuente: Fotografias Aéreas 1992, IGN Cartografia 1:50.000, Hojas: Sierpe Coronado, Térraba, IGN

Atlas Cantonal, 2014

Uso y Cobertura de la Tierra

Realizada por: Acuña, J.

Fecha del Mapa: 25-9-2015

Fig. 5. Uso y cobertura de la tierra en el Humedal Nacional Térraba-Sierpe, año 1992 Fig. 5. Land use and cover in the Térraba-Sierpe National Wetlands, 1992

de los usos antrópicos de la tierra se siguió desarrollando cercana a los lugares donde existen poblados. En comparación con el periodo anterior tuvieron un particular incremento acelerado en sus extensiones los pastos, el cultivo de arroz y los espacios naturales cercanos a boca Brava y Chiquita, Adjuntaderas y Cantarana. Los estanques para el cultivo de camarón se localizan cercanos a Puerto Cortés (Fig. 5).
Este incremento acelerado de los usos de la tierra se relaciona con distintos procesos socioeconómicos que se presentaron en el cantón de Osa, entre ellos la partida de la Compañía Bananera en 1984, ocasionando que gran parte de los habitantes quedaran desempleados, ya que constituía la principal actividad económica de la zona. Esto generó que se dieran nuevas actividades económicas, entre ellas surge la explotación de la madera 
de mangle para sus diferentes usos, así como el incremento de fincas en todo el cantón de Osa, donde en su mayoría fueron terrenos de pequeñas extensiones utilizados para pastos (Royo, 2009). A este contexto histórico de cambio de actividades agrarias que se dan en el cantón de Osa, se le suman las decisiones políticas del país para abordar la problemática de la partida de la bananera, se desarrolló un proceso de parcelamiento en los terrenos del humedal, el Estado costarricense, a través del Instituto de Desarrollo Agrario (IDA), otorgó espacios para cultivar dentro del humedal. Sin embargo, no existió ninguna regulación sobre las actividades a desarrollar en las parcelas y las personas fueron habitando zonas cubiertas de manglar, esto ocasionó que se dejara a un lado el objetivo con el que se les entregaron las tierras, respondiendo a las necesidades de tener un espacio donde habitar y realizar sus actividades productivas (Cedeño et al. 2010).

Otro factor que influyó durante esta época fue la menor protección del humedal, a partir de 1977 contó con la categoría de manejo Reserva Forestal. Dicha categoría daba la potestad de desarrollar algunas actividades en el humedal, entre ellas destacan el permiso de aprovechamiento forestal para obtener carbón, madera y taninos, también permitía el desarrollo agrario (Salas et al. 2012). Esto demuestra que el manejo del ecosistema para ese momento presentaba otras condiciones. Los procesos naturales relacionados con la erosión del terreno siguieron presentándose en distintas bocas del humedal como son: Brava, Chiquita y Zacate, lo cual influyó en la pérdida de la vegetación de mangle, situación similar al periodo de 19481972 (Acuña-Piedra \& QuesadaRomán, 2016).

En los siguientes 20 años, entre 1992 y 2012, la cobertura de la vegetación del humedal logró recuperar extensión, abarca 13911.73 ha entre la cobertura de mangle y las zonas pantanosas que corresponden al $84 \%$ de la cubierta del terreno. Esto indica que se dio un aumento del área de vegetación natural del humedal, se recuperaron más de 450 ha (Fig. 2). En cuanto a las actividades de usos de la tierra siguieron creciendo bajo un proceso menos expansivo a los años anteriores, aumentó 130 ha, destacando una nueva actividad agrícola, el cultivo de la palma aceitera que tenía un área de 12.64 ha, se mantiene la actividad de los pastos limpios y arbolados, aunque con una cobertura mayor a 347.98 ha. El cultivo de arroz cubría 56.20 ha, el área de estanques para maricultura crece a 263.26 ha y se dio un decrecimiento de las tierras desnudas o degradadas, ya que pasan a ocupar 1.18 ha (Fig. 6). Para este periodo se identifican lagunas costeras, siendo el segundo periodo cuando se tiene este tipo de coberturas en el humedal. La distribución de las actividades de los usos de la tierra se sigue desarrollando en los mismos sectores mencionados en el periodo anterior, con un incremento más relevante hacia la parte norte del HNTS, principalmente de pastos $\mathrm{y}$ 
arroz. La plantación de palma aceitera se presenta en las cercanías del poblado de Cantarana (Fig. 6).

La intensa actividad y crecimiento de los distintos usos de la tierra en la década de 1990 generan cambios en el último periodo de análisis, aumentan en más de 20 ha la cobertura de mangle y en 433 ha las zonas pantanosas (Fig. 6). Esto se relaciona con la normativa nacional que favoreció la protección de los ambientes fluviomarinos, entre estas, las medidas de protección para este ecosistema por medio de la declaración de Área Silvestre Protegida en la categoría de sitio humedal en el año 1994 y la otorgación internacional como sitio Ramsar en 1995 (Salas et al. 2012). Asimismo, se aprueban diferentes leyes ambientales como la Ley Orgánica del Ambiente ( $\left.\mathrm{N}^{\circ} 7554\right)$ en 1995, la cual fortalece la protección de los humedales, la Ley Forestal $\left(\mathrm{N}^{\circ}\right.$ 7575) en 1996, la cual establece que debe eliminarse el aprovechamiento del recurso forestal de los ecosistemas

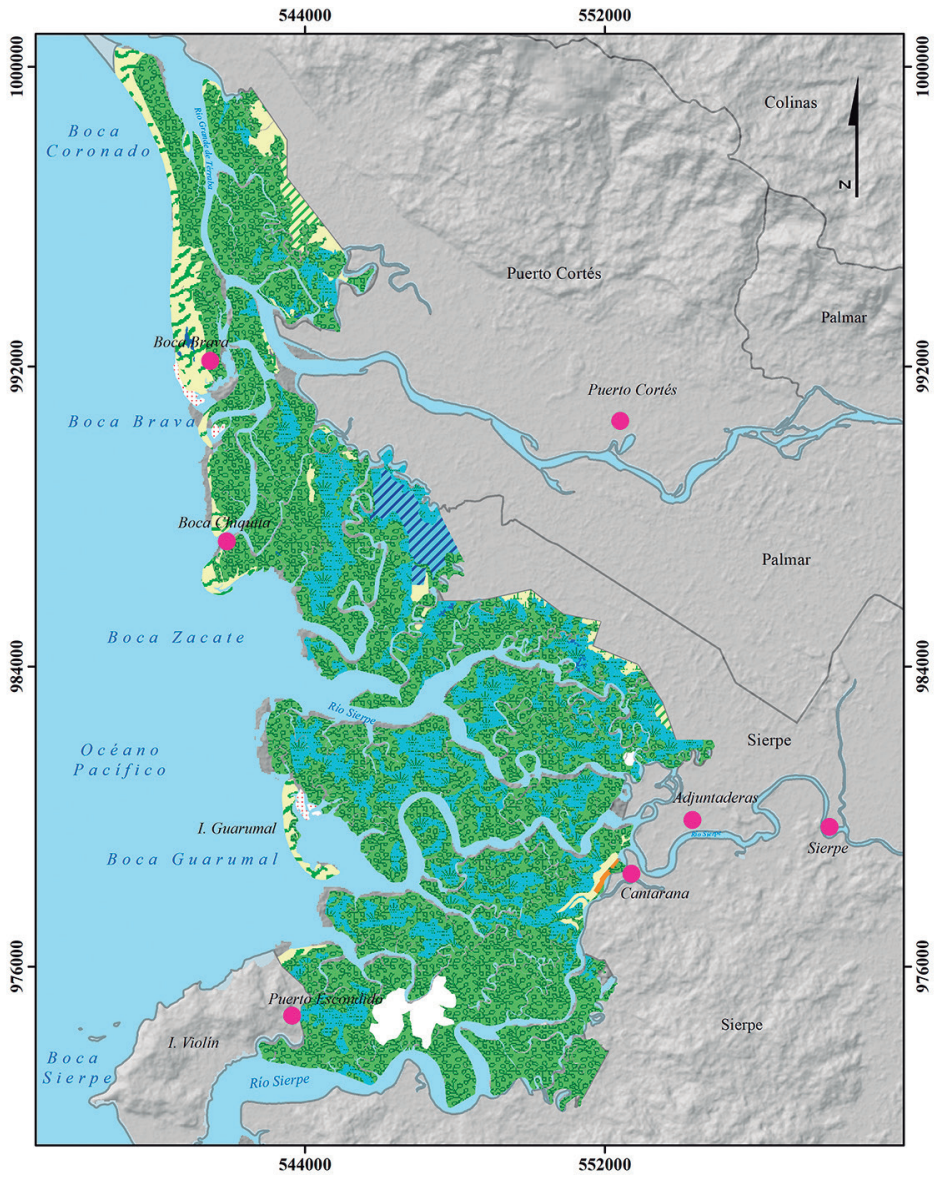

\section{Simbología}

- Poblados

Uso y Cobertura de la Tierra

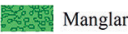

Zonas Pantanosas

X*. Pastos-Espacios Naturales

YIII/, Pastos Arbolados

Pastos Limpios

int Arroz

$\rightarrow$ Palma Aceitera

VII/, Estanque Maricultura

Laguna Costera

Tierras Desnudas-Descubiertas

Pi:i: Playas y Arenas

$\square$ Área Sin Información

Límite Distrital

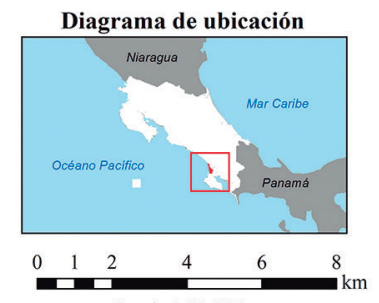

Escala 1:83.000

Proyección CRTM05

Transversal Mercator

Datum WGS 84

Fuente: Fotografias Aéreas 2012, IGN

Cartografia 1:50.000, Hojas: Sierpe

Coronado,Térraba, IGN

Atlas Cantonal, 2014

Uso y Cobertura de la Tierra

Realizada por: Acuña, J.

Fecha del Mapa: 25-9-2015

Fig. 6. Uso y cobertura de la tierra en el Humedal Nacional Térraba-Sierpe, año 2012 Fig. 6. Land use and cover in the Térraba-Sierpe National Wetlands, 2012 
de manglar que habían existido hasta ese momento (Pizarro et al. 2004), entre otros convenios internacionales.

Esta misma tendencia de disminución del área ocupada por algunos usos de la tierra se reflejó en las extensiones de pastos y terrenos degradados. No obstante, en los casos donde los usos de la tierra no disminuyen su área como son el cultivo de arroz, la palma aceitera y los estanques de maricultura, se da una tendencia de crecimiento desacelerado, en comparación a las décadas pasadas. En el año 2013 se aprueba oficializado el Plan General de Manejo del HNTS (MINAET, 2013), herramienta que contribuye al incremento en la cobertura de mangle, establece medidas de protección para este humedal, permite que se mantengan aquellas áreas donde se desarrollaron actividades de uso de la tierra, aprueba el desarrollo de la actividad turística y la pesca, propone la reducción de áreas con otros usos no naturales y la recuperación del humedal con prácticas de reforestación amigables con el medio ambiente (MINAET, 2013).

Estos procesos de protección del humedal favorecieron la recuperación del manglar, asimismo, los procesos de sedimentación de ciertas partes del delta debido a cambios en los aportes de sedimentos y salvaguarda de este ecosistema beneficiaron este aumento de cobertura de mangle, la planicie deltaica crece 613.07 ha, contribuyendo a los procesos naturales de una nueva colonización de manglar (Acuña-Piedra \& Quesada-Román, 2016). Otros factores vinculados con el incremento de la cobertura de mangle en el HNTS se relacionan con el apoyo de instituciones públicas y ONG del país, la Universidad de Costa Rica (UCR) realizó capacitaciones a la población del humedal (Forester \& Arroyo, 2014), incentivando la reforestación de mangle en los años 2012 y 2013 en boca Guarumal.

\section{Análisis de deforestación del Humedal \\ Nacional Térraba-Sierpe de 1948 al 2012}

Los resultados de los análisis de deforestación de los 64 años analizados en tres periodos comparados se basaron en tres parámetros: Pérdida Periódica Neta, Pérdida Anual Neta y la Tasa de Deforestación.

En el primer periodo comparado (1948-1972) la Pérdida Periódica Neta fue de alrededor de 1458 ha y la Pérdida Anual Neta fue aproximadamente de 60 ha anuales durante los 24 años que comprenden este periodo. La Tasa de Deforestación para este periodo fue de $0.46 \%$ (Cuadro 3 ), se considera con valor medio en conformidad con Céspedes y Moreno (2010), quienes establecen para México que a partir de un $-0.5 \%$ de la tasa de deforestación pasa a ser una pérdida acelerada de cobertura forestal. Este proceso de deforestación de mangle de las décadas de 1940 a 1970 es una tendencia que se presentó en el país no solo para bosque de manglar, sino para la cobertura boscosa en general, ya que en Costa Rica se llegaron a deforestar más de 50 000 ha por año (Barrantes, 2000).

Para el segundo periodo que va de 1972 a 1992, la cifra de deforestación es mayor al periodo anterior y durante los 64 años analizados, en promedio hubo una desaparición de 77 ha por 
año, con una pérdida periódica neta de más de 1550 ha y una tasa de deforestación de 0.64\% (Cuadro 3). Esta cifra de deforestación alta también se relaciona con el proceso que enfrentó el país de pérdida acelerada de la cobertura boscosa para las décadas de 1980 y 1990, según Chavarría (2010), de 1986 a 1996 se perdieron 16448.5 ha anuales.

Por su parte, en este periodo se observa el mayor proceso de deforestación, también obedece al comportamiento que enfrentaron otros países que cuentan con este tipo de ecosistemas, según la FAO (2007) para la década de 1980 a 1990 existía una extensión de 15.6 millones de hectáreas de manglar en el mundo, para ese periodo las tasas de deforestación eran bastante significativas en Asia, el Caribe y América Latina; en la década del 2000, la deforestación disminuye, sin embargo, el problema sigue vigente en la actualidad, donde las actividades agrarias y los procesos urbanos son la principal causa de la pérdida de mangle en estas décadas.

En el tercer periodo que corresponde de 1992 al 2012, los resultados indican que hubo una recuperación de la cobertura de mangle en el HNTS, la pérdida periódica neta obtiene una ganancia de más de 450 ha y la pérdida anual neta para las dos décadas que comprende este periodo es de una ganancia de 22 ha por año. Es el único periodo donde se reportan datos positivos de la tasa de deforestación (Cuadro3).Este proceso de recuperación de las coberturas boscosas en el país se vincula con varias decisiones tomadas por el gobierno, entre la que destaca la medida de proteger la cuarta parte del territorio nacional para la conservación mediante un sistema de áreas silvestres protegidas del Estado en diferentes categorías de manejo (Barrantes, 2000), donde a su vez el Humedal Nacional Térraba-Sierpe se declara como Área Silvestre Protegida en la década de 1990 y se refleja el cambio positivo de recuperación de área de mangle en la década del 2000.

El análisis de los 64 años identificó el comportamiento en todo el periodo e indica que la pérdida periódica neta que enfrentó el HNTS fue de más de 2500 ha con una pérdida anual neta de 40 ha por año. La tasa de deforestación tuvo cifras negativas del orden de $0.30 \%$,

Cuadro 3. Resultados de pérdida periódica neta, anual y porcentaje de deforestación del bosque de mangle en el Humedal Nacional Térraba-Sierpe, de 1948 al 2012

Table 3. Results of annual net periodic loss and deforestation percentage of the mangrove forest in the Térraba Sierpe National Wetlands from 1948 to 2012

\begin{tabular}{lcccc}
\hline Deforestación/Periodos & $\mathbf{1 9 4 8 - 1 9 7 2}$ & $\mathbf{1 9 7 2 - 1 9 9 2}$ & $\mathbf{1 9 9 2 - 2 0 1 2}$ & $\mathbf{1 9 4 8 - 2 0 1 2}$ \\
\hline Pérdida Periódica Neta (ha) & -1458.51 & -1558.17 & 453.89 & -2562.479 \\
Pérdida Anual Neta (ha) & -60 & -77 & 22 & -40 \\
\% Deforestación & 0.46 & 0.64 & 0.19 & 0.30 \\
\hline
\end{tabular}


que resulta de la deforestación de mangle a lo largo de poco más de seis décadas de análisis (Cuadro 3). Otros países enfrentaron situaciones similares relacionadas con la pérdida en la cobertura vegetal en los ecosistemas de manglar, entre ellos, Madagascar en el río Mangoky donde se deforestaron alrededor de 11790 ha de 1979 al 2000 (Rakotomavo \& Fromard, 2010), la India en el delta Sundarbans en Bangladesh y Sri Lanka perdieron 16179 ha en los años 1975 al 2000 (Giri \& Long, 2014), y en el Corredor de Mangle cercano al golfo de Fonseca entre El Salvador y Nicaragua, se perdieron alrededor del $30 \%$ de las superficies originales de mangle en las últimas cuatro décadas (Alfaro, 2011).

Los manglares en América Central son unos de los ecosistemas más importantes que se encuentran en las zonas costeras protegidas en la región, todos los ecosistemas ubicados en el istmo centroamericano sirven de puentes ecológicos entre las zonas norte y sur del continente (Windevoxhel \& Imbach, 1999). Fungen como una barrera de protección de las costas contra la erosión, vientos y mareas, además son fuentes de energía en los ecosistemas marino-costeros, asimismo, se les considera refugio de vida silvestre de la región por ser lugares de alimentación y crianza de muchas especies de flora y fauna (Sánchez, 1986). Por lo tanto, el HNTS es un ecosistema único en el Pacífico sur de Centroamérica por su conservación y extensión del manglar (Murillo, 2012), a la vez cumple con importantes servicios ecosistémicos en el entorno geográfico donde se ubica y hasta para toda la población costarricense (Sánchez et al. 2013). Es por esto que todas las afectaciones que ha tenido en el contexto de deforestación y expansión agraria, han generado impactos al medio ambiente y a los usuarios que dependen de los servicios que brinda el ecosistema de manglar en el HNTS, asimismo, son necesarias su protección y restauración para preservar la alta biodiversidad presente.

\section{CONCLUSIONES}

Durante poco más de seis décadas el HNTS ha enfrentado el desarrollo de diferentes usos de la tierra, donde destacan el cultivo de arroz, los pastos con árboles y limpios, la palma aceitera, el terreno descubierto y los estanques para maricultura. Además, entre las coberturas que se identificaron están: la vegetación de mangle, áreas pantanosas, arenas y playas, así como pequeños sistemas lagunares. El cambio en el uso de la tierra a lo largo de este periodo favoreció la deforestación de amplias extensiones de la vegetación de mangle propia del delta y humedal Térraba-Sierpe.

La deforestación a lo largo del periodo de estudio fue variable a partir de la expansión de la frontera agrícola en las décadas de 1950 y 1960 . La diversificación de la agricultura hacia actividades acuícolas durante la década de 1970 y la salida de la Compañía Bananera de la zona sur de Costa Rica en 1984 marcaron la apertura de nuevos espacios productivos 
por parte del Estado costarricense comprometiendo espacios naturales frágiles, lo que aumentó la presión sobre humedales y manglares con el desarrollo de cultivos como el arroz y la palma aceitera. A partir de la década de 1990 con la declaración como Área Silvestre Protegida y sitio Ramsar, además de la implementación de un paquete de leyes ambientales en el país, el Humedal Nacional TérrabaSierpe adquiere una mayor protección que ha favorecido su recuperación.

Las variaciones en las tasas de deforestación son claras en los tres periodos de análisis, de 1948 a 1972 la pérdida periódica neta fue de alrededor de 1458 ha y la pérdida anual neta fue de 60 ha por año durante los 24 años. De 1972 a 1992, la cifra de deforestación aumentó con respecto al periodo anterior, en promedio hubo una desaparición de 77 ha por año, con una pérdida periódica neta de más de 1550 ha. De 1992 al 2012, hubo una recuperación de la cobertura de mangle en el HNTS, la pérdida periódica neta obtiene una ganancia de más de 450 ha y la pérdida anual neta tuvo una ganancia de 22 ha por año. El análisis de los 64 años identificó el comportamiento en todo el periodo e indica que la pérdida periódica neta que enfrentó en HNTS fue de 2562.79 ha con una pérdida anual neta de 40 ha por año.

Este trabajo generó una línea base en el análisis de los cambios en los usos y coberturas de la tierra en el humedal más grande del país, develó y cuantificó los impactos antrópicos causados a lo largo de más de 64 años en el manglar y zonas adyacentes, y puso en evidencia las buenas decisiones en legislación ambiental tomadas durante la década de 1990 para la protección de estos frágiles ecosistemas, de gran importancia como sumideros de carbono, hábitat para la biodiversidad marina, costera y continental, así como barrera ante los drásticos cambios en las mareas, oleajes y variaciones futuras en el nivel medio del mar.

Todos estos avances en la toma de decisiones que ha tenido el HNTS se deben seguir aplicando para lograr la restauración de la cobertura de mangle que aún falta por recuperar. Asimismo, deben contemplarse planes de protección para mitigar las consecuencias de la variabilidad climática y de las actividades antropogénicas que afectan este ecosistema, como las realizadas en la cuenca alta y media del río Grande de Térraba, entre las que destacan la expansión agraria con el cultivo extensivo de piña que genera sedimentación y contaminación por agroquímicos, los cuales debilitan la salud del ecosistema a largo plazo.

Por último, no se debe bajar la guardia en la conservación tanto del Humedal Nacional Térraba-Sierpe como de sus zonas adyacentes, ya que su recuperación no se ha dado en su totalidad. Las amenazas siempre están presentes, como en la actualidad la construcción del aeropuerto en las cercanías del HNTS y las recientes pretensiones de cultivar piña en las proximidades del manglar. Esta es una tarea de la población civil, la academia y las instituciones públicas todos los días. 


\section{BIBLIOGRAFÍA}

Acuña-Piedra, J. F. \& Quesada-Román, A. (2016). Evolución geomorfológica entre 1948 y 2012 del delta Térraba-Sierpe, Costa Rica. Cuaternario y Geomorfología, 30(3-4), 49-73. https://doi. org/10.17735/cyg.v30i3-4.53055

Alfaro, S. (2011). Cobertura y uso de la tierra en el ecosistema de mangle y zona ecotonal del corredor del mangle, desde la bahía de Jiquilisco, El Salvador, hasta el estero Padre Ramos, Jiquilillo, Nicaragua. Rev. Geogr. Am. Cent., 2(47), 1-19.

Barrantes, G. (2000). Aplicación de incentivos a la conservación de la biodiversidad en Costa Rica. San José, Costa Rica: MINAE-INBio-IPS.

Barrantes, M. B. \& Cerdas, A. (2015). Distribución espacial de las especies de mangle y su asociación con los tipos de sedimentos del sustrato, en el sector estuarino del Humedal Nacional Térraba-Sierpe, Costa Rica. Rev. Biol. Trop., 63(1), 47-60.

BIOMARCC-SINAC-GIZ. (2012). Evaluación de carbono en el Humedal Nacional Térraba-Sierpe. Proyecto Biodiversidad Marino Costera en Costa Rica, Desarrollo de Capacidades y Adaptación al Cambio Climático. San José, Costa Rica. s.e.

Cedeño, B., Hernández, A., López, A. \& Villalobos, E. (2010). Caracterización socioeconómica de la cuenca del Río Grande de Térraba. Heredia, Costa Rica: EUNA.

Céspedes, E. \& Moreno, E. (2010). Estimación del valor de la pérdida de recurso forestal y su relación con la reforestación en las entidades federativas de México. Invest. Amb., 2(2), 5-13.

Chavarría, I. (2010). Costa Rica: Algunas cifras forestales al 2010. Sistema de Información de Recurso Forestal de Costa Rica. Recuperado en septiembre 6, 2017, disponible en http://www.sirefor. go.cr/Documentos/Bosques/2010_Chavarria_Algunas_cifras_forestales.pdf

Duarte, E., Emanueli, P., Milla, F., Orellena, O. \& López, S. (2015). Análisis de cambios de la cobertura forestal y uso de la tierra mediante imágenes satelitales de alta resolución espacial: sitio piloto cantón de Puriscal años 2009-2012-2015 República de Costa Rica. San José, Costa Rica: Deutsche Gesellschaft für Internationale Zusammenarbeit (GIZ) GmbH.

ESRI. Environmental Systems Research Institute. (2010). ArcGIS 10. Redlands, California, EE. UU.: Environmental Systems Research Institute.

FAO. (2001). Causas y tendencias de la deforestación en América Latina. Programa de Evaluación de Recursos Forestales. Recuperado en mayo 25, 2017, disponible en http://www.fao. org/docrep/007/ad680s/ad680s00.htm.

FAO. (2007). Los manglares de América del Norte y de América Central 19802005. Roma, Italia: FAO.

Forester, S. \& Arroyo, D. (2014). Ecoturismo en el Humedal Nacional Térraba Sierpe: herramientas para su desarrollo. San José, Costa Rica. s.e.

Giri, L. \& Long, J. (2014). Distribution and dynamics of mangrove forests of South Asia. J. Environ. Manage., 1(146), 101-111. https://doi. org/10.1016/j.jenvman.2014.01.020

Hernández, H. (1985). Costa Rica: Evolución territorial y principales censos de población 1502-1984. San José, Costa Rica: EUNED.

Jiménez, J. (1993). Alternativas de manejo de los manglares en el contexto del Pacifico 
de Centroamérica. Ministerio de Agricultura y Ganadería. San José, Costa Rica. Recuperado en mayo 27, 2017, disponible en http://www.mag.go.cr/congreso agronomico_ix/A01-1277-21.pdf

Kappelle, M., Castro, M., Acevedo, H., González, L. \& Monge, H. (2002). Ecosistema del Área de Conservación Osa (ACOSA). Santo Domingo, Costa Rica: INBIO.

Lizano, O., Amador, J. \& Soto, R. (2001). Caracterización de manglares de Centroamérica con sensores remotos. Rev. Biol. Trop., 49(1), 331-340.

Mainardi, V. (1996). El manglar de Térraba Sierpe en Costa Rica. Turrialba, Costa Rica: CATIE.

Méndez, L. \& Guzmán, J. (2006). Ecosistema de manglar en el Archipiélago Cubano: Estudios y experiencias enfocados a su gestión. La Habana, Cuba: Editorial Académica.

MINAET. (2013). Decretan Resumen Ejecutivo del Plan de Manejo del Humedal Nacional Térraba Sierpe. (Publicado en La Gaceta $N^{\circ}$ 242, 3-11, 16 de diciembre). San José, Costa Rica: Imprenta Nacional.

Mora, F. (2013). Morfodinámica litoral de boca Zacate, su impacto en la cobertura del bosque de mangle y su relación con las actividades económicas de los habitantes de Sierpe, cantón Osa, Costa Rica: recomendaciones para una gestión integrada. Tesis de maestría no publicada, Universidad de Costa Rica, Montes de Oca, Costa Rica.

Murillo, K. (2012). El Proyecto de Aeropuerto Internacional para el Sur: Contexto, Percepciones y Perspectivas. San José, Costa Rica: Stanford Woods Institute for the Environment Stanford University.

Ortiz-Malavassi, E. (2012). Cambios geomorfológicos en el litoral Caribe y Pacífico de Costa Rica. Caso del Complejo Déltico de Sierpe. Revista Forestal Mesoamericana Kurú, 5(15), 45-54.

Pizarro, F., Piedra, L., Bravo, J., Asch, J., $\&$ Asch, C. (2004). Manual de procedimientos para el manejo de manglares en Costa Rica. Heredia, Costa Rica: EUNA. Proyecto Humedales. (2015). Humedales de Importancia Internacional de Costa Rica. Heredia, Costa Rica: SINAC/ PNUD.

Rakotomavo, A. \& Fromard, F. (2010). Dynamics of mangrove forests in the Mangoky River delta, Madagascar, under the influence of natural and human factors. Forest Ecol. Manag., 1(259), 1161-1169. https://doi.org/10.1016/j. foreco.2010.01.002

Rosales, A. (2016). Leyenda CLC-CR para la generación de/para uso/cobertura de la tierra de Costa Rica (Leyenda corine land cover versión Costa Rica v 1.0). Recursos digitales. San José, Costa Rica: MAG/INTA.

Royo, A. (2004). La ocupación del Pacífico Sur costarricense por parte de la compañía bananera (1938-1984). Diálogos: Rev. Elec. His., 4(2), 6, 1-14.

Royo, A. (2009). El supuesto determinismo de los cantones bananeros: el cantón Osa, Zona Sur, Costa Rica. Población y Salud en Mesoamérica, 6(2), 1-37. https://doi.org/10.15517/psm.v6i2.1603

Salas, E., Ross, E. \& Arias, A. (2012). Diagnóstico de áreas marinas protegidas y áreas marinas para la pesca responsable en el Pacífico costarricense. San José, Costa Rica: Fundación Mar Viva.

Salitchev, K. A. (1979). Cartografia, arquitectura y urbanismo. La Habana, Cuba: Pueblo y Educación, MES. 
Sánchez, R. (1986). Metodología descriptiva para determinar los posibles usos de las áreas de manglares y su aplicación en Coronado-Sierpe, Costa Rica. Tesis de maestría no publicada, CATIE, Turrialba, Costa Rica.

Sánchez, R., Reyes, V., Mora, R., Castro, R., Madrigal, P., Ovares, C. \& Casantes, S. (2013). Valoración económica de usos alternativos de la tierra del área de amortiguamiento y del Humedal Nacional Térraba-Sierpe (HNTS). San José, Costa Rica. s.e.

Scheffers, A. \& Browne, T. (2010). Pacific Coasts of Guatemala, El Salvador, Honduras, Nicaragua, Costa Rica and Panama. In E. Bird (Ed.), Encyclopedia of the World's Coastal Landforms (pp. 187191). New York, EE. UU.: Springer.
Vargas, E. (2007). El universo del manglar Sierpe-Térraba en Costa Rica. Traducción y cambios socio-ambientales del ecosistema estuarino. Ciudad Colón, Costa Rica: s.e.

Windevoxhel, N. \& Imbach, A. (1999). Uso sostenible de manglares en América Central. Manejo productivo de manglares en América Central. Proyecto Conservación para el Desarrollo Sostenible en América Central. Turrialba, Costa Rica: CATIE. Recuperado en mayo 27, 2017, disponible en www.bvsde.org.ni/Web_textos/GOLFONSECA/.../10Manglar\% $\% 20$ A\%20Central.pdf

Zaldívar, A., Herrera, J., Coronado, C. \& Parra, A. (2004). Estructura y productividad de los manglares en la reserva de biosfera Ría Celestún, Yucatán, México. Rev. Cient. Am. Lat. Car., Esp. Por., 10(1), 25-35. 